\title{
GESTION Y POLITICA INDUSTRIAL EN SECTORES DE ALTA TECNOLOGIA: \\ El Caso de los Semiconductores *
}

\author{
Shelby H. Carter, Jr. \\ William W. Cooper \\ George Kozmetsky \\ Kingshuk K. Sinha \\ Dee Bruce Sun
}

$\mathbf{L}$

as orientaciones del gobierno de

Estados Unidos para una "política industrial" que fueran lanzadas con bombos y platillos por la puerta grande durante el gobierno del Presidente Reagan, han estado haciendo su reingreso calladamente por la puerta falsa durante el gobiemo del Presidente Bush. Recientemente, la revista Time ha informado lo siguicnte:

"Bush y sus asesores estín cantando ahora una tonada muy diferente. Las iniciativas de politica industrial que estaban siendo passadas casi clandestinamente a través de la burocracia federal (gubernamental), de repente han sido puestas al centro de la li-

- Informe de investigación de la Oficina de In. vestigación de Negocios de la Universidad de Texas en Austin. Versión revisada del documen. to que sirviera de base para el discurso de apertura de la Segunda Conferencia Anual de la Sociedad Crina para la Administración de la Tecnologia, llevada a cabo en Taipei. Taiwan, el 4 de octubre de 1991. Traducido del inglés para esta edición por Giovanna Manosalva y Teresa Ontaneda. nea delantera, fungiendo Bush mismo de animador. La semana pasada el Presidente firmó la Ley de Computación de Alto Rendimiento (Iligh Performance Computing Act) de 1991, que autoriza a ocho organismos federales a gastar US\$ 638 millones en el desarrollo de equipo y programas (hardware y software) para una computadora "teraflop" capaz de ejecutar un billón de operaciones por segundo. Ese mismo día, NISecretariode Energia, James Watkins, anunció que los 726 (si, setecientos veintisćis) laboratorios nacionales del gobierno -instalaciones que gastaban más de US\$20 mil millones por año, principalmente en la investigación dearmamentos- estarán ahora disponibles para emprender proyectos de investigación conjuntos con el sector privado" "I?

\section{Introducción}

El propósito de este artículo es examinar lo que podrían implicar los elementos de una

1) Time, Dec. 23, 1991, p. 52. 
política de esta índole, poniendo énfasis especial en el panorama permanentemente cambiante de las modernas industrias de alta tecnología. Demostraremos que la orientación correcta debe estar dirigida a la investigación intensiva y estrechamente relacionada a los desarrollos tecnologicos, y que esta puede, como lo indica la cita precedentc, tomar la forma de subsidios gubernamentales o de colaboración con empresas privadas.

Diremos que tambićn se requiere algo más con respecto a la responsabilidad para con el público. Remontaíndonos en cl tiempo, podemos abundar en lo dicho por Adam Smith hacc casi 200 antos:

\begin{abstract}
"Aunque la gente del mismo gremio rara vez se reúne, ni siquiera para celebraciones ni para divertirse, cuando lo hacen la conversación siempre termina en una cons. piración contra el público o $\mathrm{cn}$ alguna ma. quinación para aumentar los precios. En realidad, es imposible impedir esas reuniones mediante una ley que pueda ser cumplida o que esté en concordancia con la libertad y la justicia. Pero si la ley no puede impedir que la gente del mismo ne gocio se reúna, tampreco debería hacer algo para propiciar tales reuniones; ni mucho menos hacerlas necesarias $"$ "
\end{abstract}

Dentro de la perspectiva ofrecida por Adarn Smith, uno debe preguntarse qué fue lo que sucedió durante la reciente visita del Presidente Bush al Japón, en el transcurso de la cual ambos gobicmos no sólo alentaron a los líderes empresariales de sus respectivos paises a que se reunieran. sino también a que negociaran convenios similares a carteles y llegaran a otros entendimientos extraoficiales.

2) The wealth of nations, New York. The Modem L.jbrary, 1937, p. 128.
¡Esto es suficiente respecto de las facilidades que otorga el gobierno a los intereses empresariales! También es necesario ser cauteloso con las indicaciones para cumplir las regulaciones gubernamentales, incluyendo las restricciones comerciaIes. Dado el ritmo de los cambios que estaremosconsiderando, en el mejor de los casos tales regulacioncs posiblemente quedarán obsoletas antes que puedan ser negociadas (y legisladas) y, por lo tanto, probablemente hagan más mal que bien cuando entren en contacto con los escenarios rápidamente cambiantes de la industria moderna. Advertencias similares se aplican a la creación de nuevos estratos de burocracias engorrosas, como lo indica la siguiente observación del Hudson Institute (citado en el mismo artículo de Time antes mencionado):

TEste| grupo de expertos conservado. ros [el Hudson Institute] propone que Washington cree un nuevo organismo federal para la ciencia y la tecnología con el propósito de coordinar la inversión tan dispersa del gobiemo [de los Estados Uni. dos] en I\&D [investigación y desarrollo]. que asciende a US5 76 mil millones anua. les, y que convierta a la industria en un socio a todo nivel".

Casi con toda seguridad, un organismo de esta índole se convertiria en el centro de intereses creados y grupos de presión, que lo inducirían a comportarse de manera análoga a como lo ha hecho el Departamento de Educación đe Estados Unidos al ajustarse al statu quo desde que el Presidente Carter le concedió rango ministerial en 1979. Es posible que, tanto en su aspecto político como en su aspecto administrativo, un nuevo organismo de I\&D de este tipo se convierta cn una fuerna estabilizadora y defensora del statu quo cuando se necesita precisamente 
lo contrario, ahora que Estados Unidos se va adaptando al hecho de dejar de lado la Gucrra Fría.

Tal como veremos, el cambio y la respuesta flexible a lo que está ocurriendo son los ingredientes necesarios de una política industrial dirigida a atender los requerimientos de la industria de alta tecnología. Con el fin de enfocar todo esto de una manera más clara, aquí nos concentraremos en las empresas de semiconductores para ilustrar lo que sectores como éste implican para la política pública. Aunque los semiconductores sólo son una parte del cuadro total, sus problemas tipifican los escenarios cambiantes a los cuales deben enfrentarse las industrias de alta tecnologia. Andrew S. Grove (presidente y gerente general de Intel Corporation) cxpone correctamente los problcmas por solucionar en su respuesta a un extenso debate publicado en un número de llarvard Business Review:

"Así como no se puede construir automóviles de carrera de fórmula uno con pieras de inventario, tampoco se puede desarro. Ular microprocesadores de alto rendimiento con las tecnologías genéricas de los semiconductores. Es necesario afinat la microarquitectura de una arquitectura avanzada a la tecnología bísica del silicio, y la tecnología del silicio a la microarquitectura para lograr un máxirno rendimien. to. Tales esfuernos de optimización se extienden tambión a los instrumentos de diserio, empaque y prueba" $y$.

En resumen, se trata de una situación de cambio continuo y de interacción en formas complejas cntre todas las fases de los productos y los procesos de estas industrias.

3/ Harwand Business Review, Sep-Oct., 1991, p. 141.

\section{Complejidad y cambio en la fabri- cación de semiconductores}

Chen, Harrison, Ackere y Wein ${ }^{2}$ han señalado que la fabricación de semiconductores es una de las operaciones más complejas de producción. Tal como se indica en la siguiente caracterización de lo que está sucediendo, se enfrenta también a serios desafios, pues el incremen. (t) de la capacidad de sus productos se ha visto acompanado por una rápida declinación de sus precios.

"Si los fabricantes de automóviles hubieran mejorado sus tecnologias tanto como los fabricantes de semiconductores, un Rolls Royee costaria ahora USS 3 y ren. diría 100 millones de millas por galón".

Esta cita corresponde a George $\mathrm{H}$. Heilmeier s", presidente de Bell Communieations, Inc. (Bellcore), empresa que desputs del desmembramiento de AT\&T (de conformidad con la Ley Anti Monopolio de Estados Unidos) se convirtió cn la sucesora de los ex Bell Labs de AT\&T. Según lo describe John Bardeen". coinventor del transistor (con William Shockley y Walter Brattain), en la dócada de 1940 los Bell Laboratories de AT\& $\mathrm{T}$ deben haber proporcionado un marco extraordinariamente alentador para estos desarrollos. Queda por ver si Bellcore estará a la altura del desafio que significa

4/ II. B. Chen, J.M. Ilarrison, A. Ackere y L. M. Wein, "Queveing network models of semiconductor wafer fabricatice". Technical Report, Center for Integrated Systems, Stanford University, 1986.

5/ Business Week, Jul. 29, 1991, p. 34.

6) John Baedeen, "To a solid state", Science, Nuv. 1984, pp. 147.145. 
continuar el récord de su predecesor, pero desde nuestro punto de vista, queremos recalcarlo, fue el trabajo cmanado de este laboratorio el que sirvió de trampolín a los subsiguientes desarrollos.

No obstante, incluso la declaración đel Dr. Heilmeier, quizá đemasiado graífi$\mathrm{ca}$, omite manifestar lo que se ha logrado en miniaturización y lo que se ha debido enfrentar por causa de la creciente complejidad de la fabricación de semiconductores. Prosiguiendo a un ritmo al cual, según John Bardecn", durante los últimos años el número de componentes por chip se ha más que duplicado de año en aflo, el proceso continúa yendo de prisa -como lo atestigua la exitosa carga de más de un millón de componentes en un chip más pequeño que la uña de un dedo de la mano-. Y, tal como to informara Business Week, aparentemente nos estamos encaminando hacia un futuro con quizá miles de millones de componentes contenidos en un chip más pequeno que el diámetro de un cabello humano".

Desarrollos como éste obligaron a la Comisión de Estudios de la ONU a usar el número de componentes por chip para obtencr una descripción real de lo que estaba sucediendo en la producción de

7) Otros han usado tasas de incremento más moderadas, pero sicmpre muy altas. Véase, por ejemplo, J. A. Fisher y B. A. Rau "Instructionlevel parallel processing", Science, Set., 13, 1991. p. 1233, quienes estiman que: "el número de transistores en un microprocesador ha aumentado aproximadamente a un ritmo de 40 por ciento por afio, y esta tendencia no muestra signos de ceder".

8/ "A big step toward the billion transistor chip". Buriness Week, Jun. 3. 1991, p. 124. scmiconductores $*$, Según esta descripción, lo que interesa en la producción es el número de componentes en vez del número de chips. Sin embargo, es inadecuadỏ tomar en cuenta sólo el creciente número de componentes por chip, pues, entre otras cosas, se omite lo que ha estado sucediendo con los procesos, los cuales también han estado experimentando cambios. Por ejemplo, considcremos la siguiente cita de un número de Barron's publicado en 1990:

\begin{abstract}
"La industria de semiconductores está hambrienta por nuevos equipos que se vuelvan cada vez más importantes conforme los fabricantes van cargando más ele. mentos en un chip [...]. Conforme se apiffa más y más memoria en el mismo espacio. el ancho de las líneas se hace más pequeño, el número de capas se hace cada vez mayor y el potencial de error va crecien. do [.... I. Deben poder decir lo que está sucediendo en el proceso de producción de semiconductores: pero hacetlo a la antigua, con microscopio y el ojo humano. simplemente no es lo suficientemente bueno para líneas ahora cercanas a los 0.5 micrones [o millonesimas de metro]. Tienen qque automatizar cada vez más el proceso con dispositivos cada vez más sofisticados [y complejos]" it.
\end{abstract}

Se requiere algo más que un conteo del número de componentes por chip si se va a tomar en cuenta la creciente com. plejidad de los procesos asi como la de los productos. Lo mismo sucede con los productos que se estain fabricando. Es cl número de relaciones posibles entre componentes el que se asocia con un mayor

9/ Transmational corporations in the international semicanductor industry, New York, The United Nations, 1986.

1Q Barron's, Sep. 24,1990, p. 18. 
rendimicnto, y estas posibilidades correlativas aumentan combinatoriamente en vez de sólo linealmente con el número de componentes. Para dar cierta idea de lo que esto significa, en la Figura 1 presentamos algunos de los resultados de un estudio realizado en una planta de fabricación de semiconductores, y en la Figura 2 demostramos la forma cómo estos resultados podrían ser empleados en actividades de planeamiento estratégico.

La curva que aparece en la parte inferior de la Figura 1 representa la producción mensual ${ }^{11 /}$ de una planta durante un periodo de 28 meses. Esta curva, denominada TND (Total Net Die), indica el número de "dice" (chips integrados) producidos. La curva superior indica lo que succde cuando TND es recmplazada por otra medida de volumen, TNDE (Total NetDie Equivalent), ajustada a las variaciones de complejidad de los productos fabricados y de los procesos usados durante el periodo. Tal como puede apreciarse, la curva superior presenta una imagen diferente del producto al tomar en cuenta la mayor complejidad de los productos y procesos correspondientes. Pero todo esto queda oculto a la vista cuando se representa sólo un simple conteo de los chips producidos, tal como sucede en la curva inferior.

En este estudio definimos la complejidad de una manera diferente a comola considera el antes citado informe de la Comisión de la ONU. Aquí es definida (y medida) tomando como referencia el número de relaciones entre elementos (com. ponentes) así comoel número de clemen-

11/ En realidad, el periodo de los informes ha sido cstandarizado a intervalos de 4 semanas. tos contenidos en un chip, y se usa una definición comparable para reflejar la complejidad de los más de 160 procesos usados en esta planta ${ }^{12}$. Es posible entonces derivar estimados de los costos unitarios para usarlos con las medidas TNDE con el fin de obtener descripciones como la que aparece en la Figura 2. En esta figura, $\mathrm{CP}(\mathrm{A})$ muestra el nivel actual de los costos unitarios o más bien lo que serian a los niveles de "mejor desempeño" propios de la planta ${ }^{13}$, y $\mathrm{Cl}(\mathrm{A})$ muestra lo que scrían estos costos si los tiempos y rendimientos del ciclo alcanzaran el nivel de los "competidores de mejor desempeño".

Evidentemente se necesita una mejora, pero jugar simplemente a "alcanzar" noes suficiente. Se deben tomar en cuenta los cambios en los productos y en los procesos que están ocurriendo en la industria y en la empresa. El uso de extrapolacioncs que permiten el cambio

12) W. W. Cooper, K. K. Sinhay R. S. Sultivan, "Measuring the complexity in high technology manufacturing: indexes for evaluation", Interfaces (por publicarse en 1992). Se puede encontrar el detalle cumpleto con ampliaciones y elaboraciones en K. K. Sinha, "Models for evaluation of complex technological systems: strategic applications in high technology manufacturing", Ph.D. thesis, University of Texas, Graduatc School of Business, 1991, la que se podrá obiener en 1992 de University Miceofilms, Inc, 300 N. \%eeb Road, Ann Arbor. Michigan 48106 .

13/ Estos ajustes de "mejor prictica" se obtienen usando el Data Envelopment Analysis (DEA). tal como se describe en Sinha, $O p$. cit . Véase un tratamiento introductorio del DFA en R. D. Banker, A. Chames, W. W. Cooper, J. Swants y D. A. Thoenas, "An introduction to data envelopment analysis with some of its models and their uses", Research in Governmental and Nonprofit Accownting. 5, 1989, pp. 125-163. 


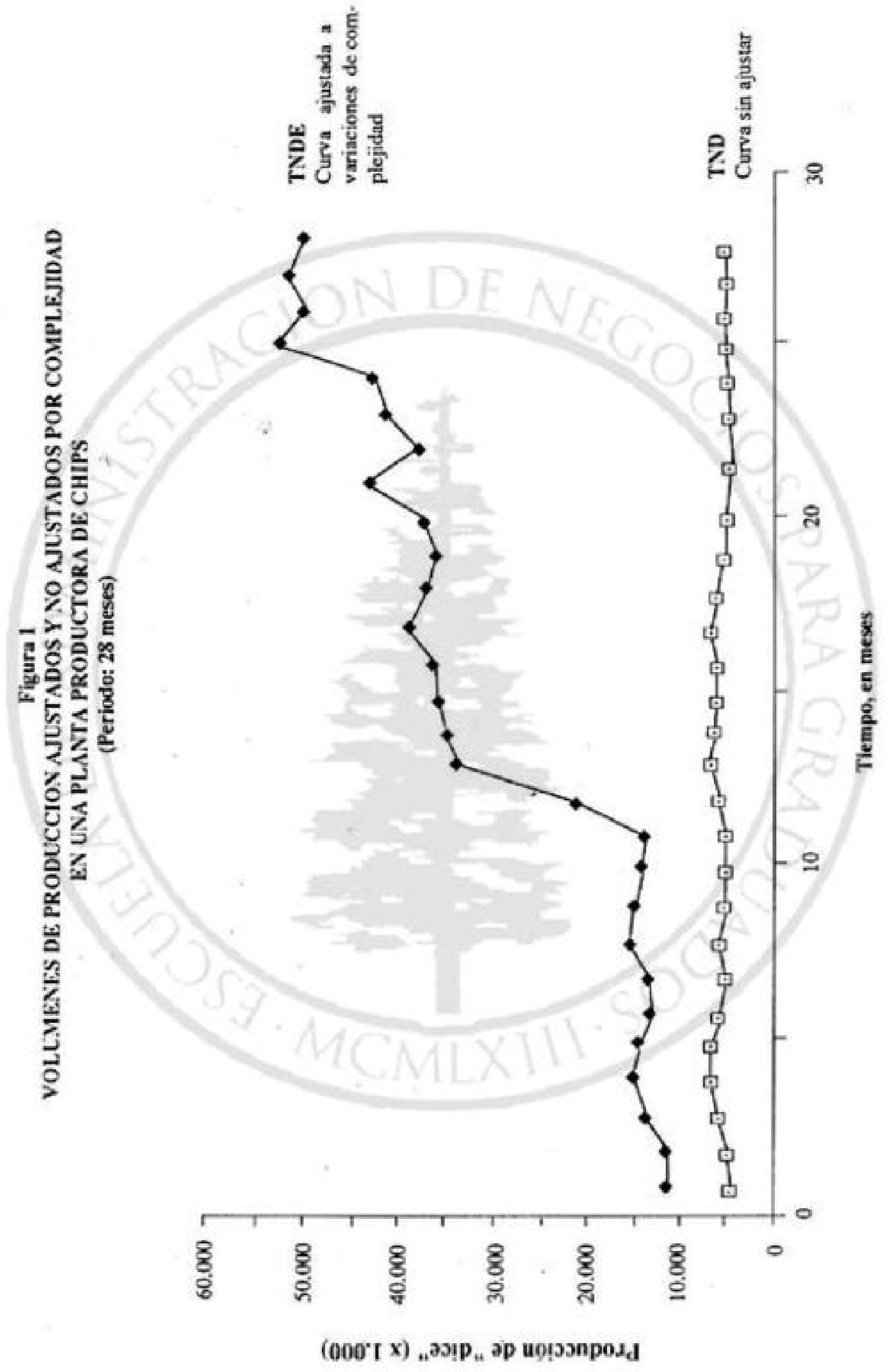




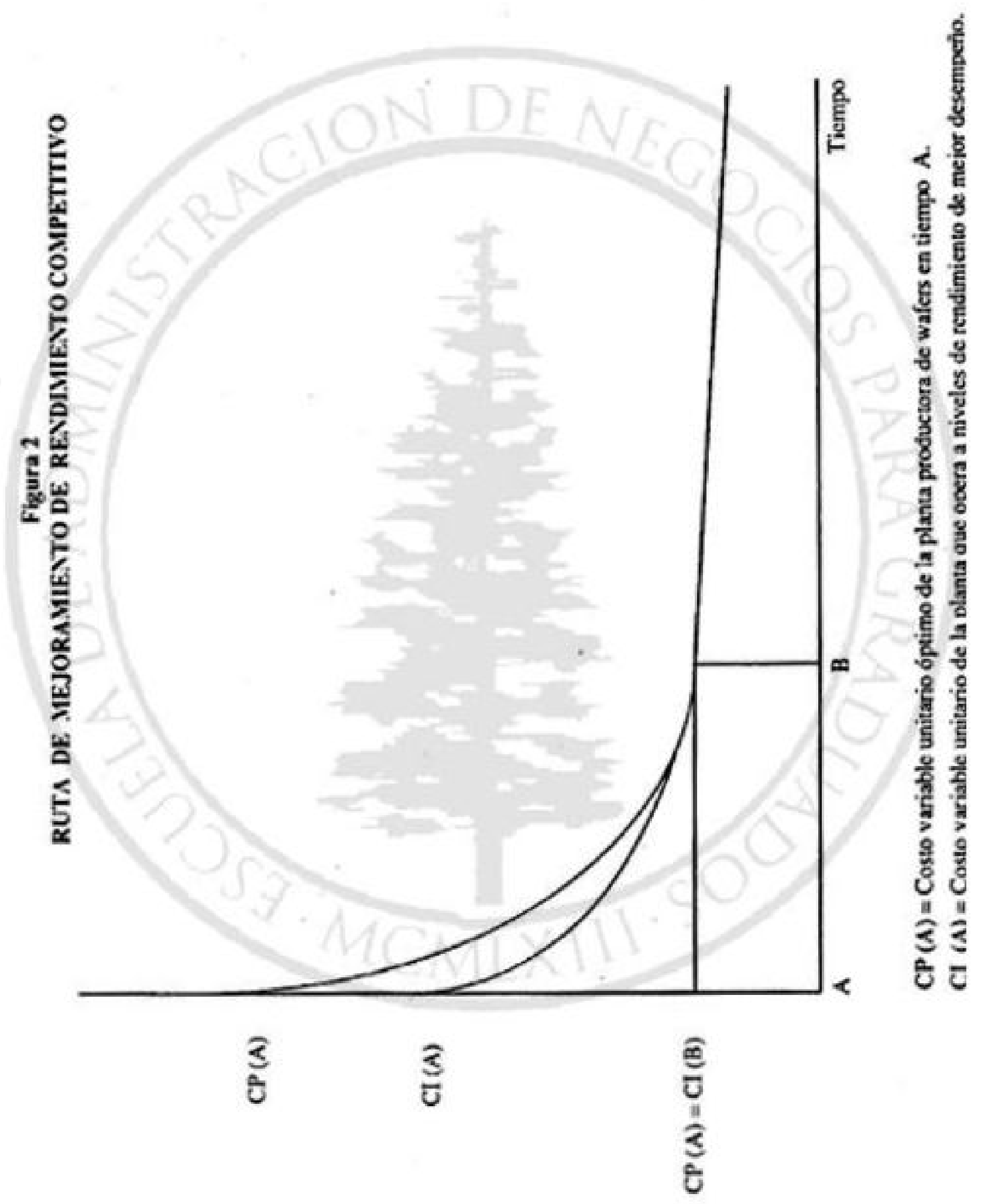


de fronteras, tal como son declaradas en términos de TNDE, tanto para la planta comopara los competidores de mejor desempeño, produce una intersección en B, tal como lo indica $\mathrm{CP}(\mathrm{A})=\mathrm{Cl}(\mathrm{B})$, En esta comparación del desempeño entre la planta y sus competidores, es tarea de la gerencia considerar si el tiempo implicado para llegar a la intersección es o no satisfactorio. Si no lo fuera, se requerirá la reconsideración de los planes actuales y el examen de lo que se puede hacer para cambiar la trayectoria actual de la planta y obtener un tiempo de intersección más corto.

\section{Competenciasuperintensiva(cutthroat competition)}

La Figura 3 ayuda a aclarar conceptualmente algunos de los problemas que pensamos deben ser considerados por la política cconómica nacional (e internacional), aun cuando en la actualidad no se disponga de datos fidedignos. Esta figura presenta el caso de una "competencia superintensiva" y los problemas que de ella se derivan, hecho conocido desde hace mucho tiempo por la tcoría económica, no obstante haber sidoescasamente contemplado en el tipo de aplicaciones de política como las consideradas aquí.

Lo tratado aquí quizá se pueda explicar mejor en términos de una célebre controversia suscitada hace algún tiempo y que salió a la luz en una nota a pie de página en un artículo del renombrado economista Jacob Viner, publicado en $1931^{14}$. Viner se quejaba de la renuencia de su

14/ Jacob Viner, "Cost curves and supply curves", Zeitschrift Für Nationalockonomic. Reimpreso en American Econcmic Association, Readings in price theory, Homewood, 11., Irwin, 1952. dibujante a comprometer sus principios matemáticos para acatar su solicitud de trazar la envolvente correspondiente a la curva de costo promedio de largo plazo (CPLP) de la Figura 3 de forma que tocara los puntos mínimos de cada una de las curvas de costo promedio de corto plazo (CPCP). Posiblemente las matemáticas de Viner no hayan sido perfectas, pero su razonamicnto económico sí lo cra, pues conocía bien las consecuencias potencialmente graves que el no tocar estos mínimos acarrearía para la permanencia de la competencia perfecta de conformidad con las leyes y políticas antimonopolio y otras similares de gobiemo de Estados Unidos, que se supone debían protegerla. Paul Samuclson describe muy bien estas consecuencias en su libro de texto introductorio a la Fconomia:

"Si el costo marginal está cayendo tual como lo muestra CMgLP en la Figura 31. la empresa tiene toda la razón para ampliar su producción, y cada nuevo paso le produce algún ingreso extra y también un menor costo extra [...] tEsto demuestra cómo y por qué la competencia falla! La tecnolo. gía se vuclve más complicada y se logra una producción eficiente sólo a una escala gigantesca [...] Por lo tanto, las empresas compctitivas tienden a ampliar su pro. ducción. Puede seguir un periodo de competencia superintensiva o destructiva durante el cual todas las empresas sufran pérdidas. Finalmente, las empresas que tienen más recursos ly están dispuestas a compromelerse] sacarín fuera a las demás, dejándonos asi sin competencia perfecta"15?

15) P. A. Sanuelson, Economics: an iniroductory analysis, 4th edition, New York, MeGnaw-Ilill, 1958. 
Gestion y polttica industrial en sectores de alta tecnologia

17

Figura 3 COMPETENCIA SUPERINTENSIVA

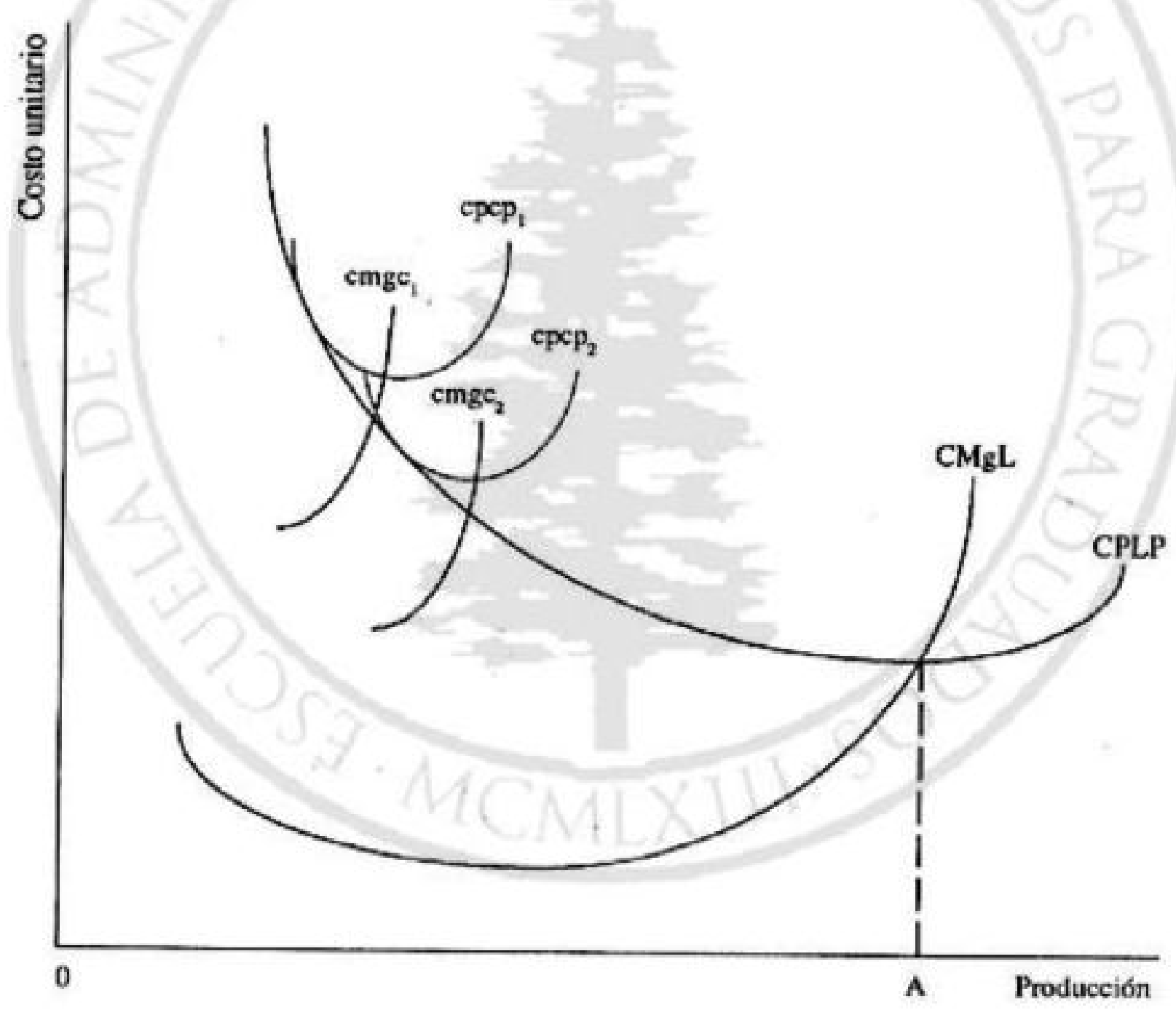


De acuerdo con los postulados clásicos de la Economía, en esta descripción Samuelson supone una tecnologia invariable y una producción homogénea. Sin embargo, esto no se aplica a la industria de semiconductores considerada aquí. Se necesita algo parecido a la medida de complejidad ajustada TNDE si descamos representar empíricamente lo que estí ocurriendo en los procesos de fabricación de semiconductores y en los productos mismos. Aqui, para nuestros fines, suponemos que todo esto se ha hecho en un estudio cuyo resultado describe un comportamiento de la industria como el representado en la Figura 3.

Existe considerable evidencia, tanto anecdótica como de otra índole, que esa competencia superintensiva ba ocurrido (y continúa ocurriendo) en la fabricación de semiconductores. Por ejemplo, Ruefli y otros realizaron un estudio sobre el periodo 1967-1986 $\mathrm{c}$ informaron haber obtenido evidencia de dumping por parte de empresas japonesas durante ese periodo ${ }^{16}$. En términos técnicos, ocurredumping cuando una empresa (extranjera) vende su producto por debajo del costo de producción. Pero esta situación se confunde fácilmente con la representada en la Figura 3, en la que las ventas por debajo del costo, e incluso por debajo del costo variable, ocurren principalmente como resultado de la estructura de una industria.

16) Timothy W. Ruefli, George Kozmetskyy Piyu Yue, "International competition in the microelectronic industry: identification of industry strategies" in T. W. Ruefli (editor), Ondinal time series analysis, New York, Quonum Books, 1990.
Los intentos por solucionar los problemas en cuestión mediante convenios, como el convenio comercial de 1986 entre Estados Unidos y el Japón, han creado aun más problemas, como lo atestigua cl siguiente editorial publicado en Forbes:

"L.Ástima que Estados Unidos renovara su convenio comercial sobre semiconductores con el Japón. El converio negociado en 1986 fue un desastre. El pacto tenía por fin detener los precios supuestamente depredatorios de Japón y ofrecer a Estados Unidos una demostración dei mereado japonés de chips. Lo que sucedió en realidad fue que los arreglos para la determinación de precios sirvieron para sacar de apuros a los fabricantes japoneses de chips al convertir su río de pérdidas (estaban sufriendo de exceso de capacidad) en un lindo pozo de utilidades. El convenio contribuyó también a producir una escasez de chips que perjudico a las empresas de computadoras y sofiware estadounidenses e hizo un flaco servicio a los consumidores, que debieron pagar precios más altos por los productos de computadoras" 17 .

La fijación de precios se hace depredatoria cuando éstos se rebajan durante un periodo para sacar del mercado a un competidor, y luego se clevan nuevamente en un periodo posterior para explotar la situación (más o menos) monopolica resultante. En efecto, parte de la conclusión de la Comisión de Comercio Internacional de Estados Unidos fue que el dumping japonés estaba resultando "amenazador" para la industria de Estados Unidos precisamente de esta mane$\mathrm{ra}^{12 /}$. Sin embargo, esta comisión no ha

Farbes, Jun. 24, 1991, p. 24. 
considerado la clase de situación representada en la Figura 3, en la cual los costos continúan cayendo conforme la producción continúa incrementándose con la aparición concomitante de productos nuevos producidos mediante procesos nuevos. En todo caso, no parece haber evidencia sustancial de precios depredatorios con la intención de aumentar los precios subsiguientemente. Lo cicrto es que los precios y los costos unitarios han seguido cayendo, exceptodurante un breve periodo de ajuste a este "convenio sobre semiconductores" japonés-estadounidense.

Tal como fue negociado por la Representante Comercial Especial de Estados Unidos, el convenio requería que los japoneses: "dejaran de vender sus productos, tanto en Estados Unidos como en paises del tercer mundo, por debajo del costo de produccióno del valor justo en el mercado del Japón"1". Esta es otra forma de dumping, es decir, vender por debajo del costo en mercados extranjeros con el fin de evitar reducciones de precios en los mercados del propio país (u otros paises). Sin embargo, tal como otros han manifestado: "es muy posible que el mercado japonés de semiconductores sea el mercadomás competitivo đel

18/ United States Intemational Commission, "Erasable programmable read ealy memories from Japan", Publication NV1778, Washington D.C., Jan., 1985. Tambitn: United States Intemational Trade Commission, "Dynamic random access memory semiconductors of 256 kilobits and above from Japan", Publication NV1803, Wastington D.C., Jan., 1986.

19/ Citado del Wall Street Journal, Apr. 6, 1987. 79 (66), p. 25. mundo"20\% . En efecto, los costos y los precios unitarios continúan cayendo en el Japón así como en el resto del mundo, cuando se permiten mejoras en los productos y las capacidades de rendimiento relacionadas con la mayor complejidad incorporada a nuestra medida TNDE.

Tal como se mencionó en la cita anterior (de Samuelson), en la Economía clásica todos los problemas se resuelven finalmente y se logra un equilibrio estable una vez alcanzado un nivel deproducción como el de A en la Figura 3. Pero según avances posteriores de la teoría económica, tal como ha observado Samuelson (en correspondencia privada con los autores), se puede esperar otro posible resultadoen la forma de distintos grados de competencia imperfecta cuando algunas empresas se esfuercen en fabricar productos diferenciados que les permitan conseguir barreras protectoras en la forma de nichosa ocupar. Sin embargo, incluso esta situación de diferenciación de produc. tos, tal como lo advierte Samuelson, debe ser modificada. Da prueba de ello, por ejemplo,el siguiente párrafo de un artículo aparecido en Business Week bajo el título de "Felicitaciones, es un Clon":

"La gente de Advanced Micro Devices Inc. sabian desde el verano pasado que el monopolio đe Intel Corp. sobre los chips 386. el mieroprocesador de mayor venta en el mundo, habia terminado [...]. Usando un enfoque de "ingeniería a la inversa" -desarmando pieza por pieza el diseño de Intel-, dos equipos [de ingenieros AMD] trabajando independientemente reconstruyerony mejoraton el sistema de circuitos,

20y Citado de S. Saba, The U.S. and japanese electronies industries: competition and cooperation",Issues in Scienceand Technology, Spr. 1986, p. 57. 
y a fines de marzo [de 1991] dieron a conocer el nuevo chip de AMD como el AM 386, que pas6 sus pruebas iniciales con todo éxito [...]. El disento de AMD funciona más rípidamente $\mathrm{y}$ procesa señales 40 millones de veces por segundo $[40$ megahertz] en comparación con los 33 MIIZ de Intel...".

Intel ha reaccionado de diversas formas contra este asalto a su nicho. Se han entablado demandas y contrademandas e incluso un organismo estatal (U.S. Federal Trade Commission) ha puesto en marcha una investigación para estudiar si Intel y otras empresas han empleado "prícticas anticompetitivas". En cfecto, parece que está por iniciarse una "guerra de precios" en toda la industria-si es que eso es una novedad-, tal como informó el Austin-American Statesman:

"En abril se iniciará una reducción de has. ta 35 por ciento en los precios de los microprocesadores 386 de Intel, el "cerebro" de la mayorfa de computadoras personales, un año después que Advanced Micro Devices Inc. rompicra el monopolio de Intel con una línea de microprocesadores rivales [...]. Seguin el plande recorte de precios, elchip 386 DX-25 de Intel se venderá por USS 99 en ver de USS 125, y su 386 XW bajará su jrecio de US5 82.50 a USS $59^{\mathrm{maW}}$.

Y, por supuesto, éste no es el final, pues tal como este mismo Austin Ame. rican Statesman continúa informando:

Un vocero de AMD dijo asimismo que la empresa proyecta reducir drásticamente los precios de su linea 386 de microprocesadores, los cuales en la mayoría de casos son algo más potentes que los de Intel. Apenas después de nueve

21/ Austin-American Statesman, Jan. 9, 1992. meses en el mercado [nicho] de los 386, AMD representa el 30 por ciento de to dos los microprocesadores 386 vendidos [...]. [Además], se prevé que a fines de enero AMD anuncie que ha desarrollado también un rival para los microprocesadores más potentes de Intel, los del tipo $486 . .$.

A parentemente, los conceptos de equilibrio de la Economía necesitan ser modificados. La dirección del cambio se desprende de esta misma nota periodistica, según la cual Intel está incrementando su presupuesto de I\&D de 1,5 a 2 mil millones de dólares, y se espera que AMD ( $y$ otras) respondan de la misma manera. En resumen, la búsqueda de nichos continúa extendiéndose, pero se espera que la protección que éstos ofrecen sea transitoria $-y$ breve-.

Es evidente que podemos continuar guiándonos por los conceptos cconómicos expresados en la Figura 3, siempre y cuando los modifiquemos adecuadamente para tomar en cuenta la dinámica inherente a estas corrientes continuas de desarrollo de productos nuevos ( $y$ procesos nuevos). En este caso también se requiere algo como nuestra medida de complejidad propuesta. Al igual que en las Figuras 1 y 2 , una medida de volumen ajustada a la complejidad tiene por fin reflejar las mayores capacidades de los productos nuevos.

\section{Conclusiones}

Tal como hemos señalado, el comportamiento de industrias como la descrita se presta a malas interpretaciones y puede conducir a aplicar políticas erróneas s: sólo se considera estáticamente lo que ha bitualmente se da por sentado. Este com 
portamiento se presta, por ejemplo, para "vender por debajo del costo", pero por razones sustancialmente distintas a las generalmente asociadas con el dumping. Eistc comportamiento también es "anticompetitivo", no en el sentido usual, sino, más bien, porque: "es una competencia tan fuerte que destruye la competencia", Al ser inherente a la naturaleza misma de la industria, no es la clase de comportamiento que se preste al uso directo de correctivos como pueden ser las leyes y los arreglos institucionales actuales.

Como señalamos al inicio de este trabajo, parte de lo que se necesita en cuanto a reorientaciones de política está siendo considerado ahora por la rama cjecutiva del gobierno del Presidente Bush. También está haciendo lo suyo el Congreso de Estados Unidos, el cual, scgún Paul Magnusson $^{22}$, está dispuesto a ponerle remedio con la Ley Nacional de Producción Cooperativa (National Cooperative Production Act). Esta ley, tal como ha sido propuesta, climinaria las acciones judiciales del gobierno sancionadas por triple daño, que ahora están permitidas de. conformidad con las leyes antimonopolio actuales.

Pcrmitiría también la creación de cmpresas conjuntas -incluso estadounidense-japonesas-, como las que ya sc cstán poniendo en marcha en respuesta a la carga financiera que significa fabricar chips de memoria avanzados, ahora próxima a los mil millones de dólares.

La política actual, por lo tanto, parece cstar orientada hacia desarrollos análo-

22/ Buciness Week, Jul. 29. 1991. gos en la práctica a los descritos al inicio de estc trabajo en relación con la visita que hiciera el Presidente Bush al Japón. Nucstra propia orientación se inclinaría más hacia la investigación, o a la I\&D, siempre y cuando estas actividades estuvicran acompafladas por diversas salvaguardas. En este sentido, y en conformidad con estos lineamientos, ya se cstán realizando desarrollos en la tecnología de producción de chips, tales como los de MCC (Micro-electronics \& Computer Technololgy Corp.) y la firma de investigación Sematech, ambos consorcios entre $\mathrm{el}$ gobierno $\mathrm{y}$ la industria privada que operan desde Austin, Texas, con exenciones legalmente autorizadas por las leyes antimonopolio de Estados Unidos. Próximamente es posibic que también se convierta en ley un proyecto de ley extendiendo estas excnciones a otras industrias. Probablemente estos esfuerzos de cooperación para la investigación no se limiten a la escena cstadounidense y, en efecto, Sematceh ha suscrito recientemente un convenio para compartir información con JESSI (Joint European Submicron Silicon Institute), cuya scde está en Munich, con las consiguientes discusiones que podrian ayudar a coordinar sus esfucrzos de investigación. Finalmente, la oferta, mencionada en nuestra sección inicial, que hiciera el Secretario Watkins para trabajar en colaboración con la cmpresa privada en varios cientos de laboratorios estatales de investigación puede acrecentar mucho (y probablemente acrecentarai) cstos esfuerzos.

Consideramos como objetivo prioritario salvaguardar los intereses del público. La clección o selección de representantes públicos para integrar los directorios de estas organizaciones podría constituir un medio para hacerlo, sicmpre y 
cuando se encontrara alguna forma para garantizar que los representantes públicos así designados conozcan bien los problemas altamente técnicos involucrados en estos esfuerzos.

Otra posibilidad sería el uso de "auditorias de desempeno" (performance audits) conducidas por profesionales competentes $\mathrm{e}$ independientes, cuyas conclusiones y recomendaciones se hicieran públicas. Téngase en cuenta que no estamos limitando esta actividad a las acostumbradas auditorías (que dan fe), nos estamos refiriendo más bien a las denominadas "auditorías integrales", en las cuales un auditor supone que todas las árcas de actividad administrativa son susceptibles de ser auditadas y asume la responsabilidad de elegir el área en la cual se hará la auditoría así como la audiencia a la cual dirigirá sus informes ${ }^{2 N}$.

Ađemás, ya existe una institución apropiada: la General Accounting Office de Estados Unidos, con una larga experiencia en auditorías de este tipo en una amplia gama de actividades. Goza de la necesaria independencia de la rama ejecutiva y parece poseer el profesionalismo sobre el cual basar la nucva responsabilidad que le estamos señalando, tal como lo atestigua el hecho de haberse hecho cargo de auditorías en áreas tan disímiles como las actividades de organismos de inteligencia, de diseño y operación de plantas nucleares y de producción de armamentos ${ }^{24}$.

23/ Para una exposición sinóptica véase "andits", tal como está definida en el Kohler's dietionary for accountants, 6th Edition, Englewood Cliffs, NJ, Prentice Hall, Inc., 1983.

24/ Se puede encontrar una buena muestra de la gama contemporinea de actividades de auditoria
Otro medio de asegurar niveles deseables de confiabilidad o, por lo menos, de control flexible sería el extender las fuerzas de competencia global ya descritas. Una expansión así pondría en juego a las fuerzas del mereado, en las cuales se pueđe confiar sin algo más que una supervisión general. Para esto ya existen también instituciones adecuadas, tales como la Comisión Intemacional de Comercio (International Trade Commission) y el Departamento de Justicia Federal (U.S. Justice Department). De este modo tampoco se requiere implementar nuevos departamentos u organismos antes de poder comenzar a funcionar. Más bien, lo que se tiene que hacer es establecer diferencias siguiendo los lineamientos sugeridos por la Figura 3, con el fin de complementar las doctrinas recibidas, tales como el comportamiento anticompctitivo $\mathrm{y}$ el dumping.

No es pues de sorprender que hayan estado ocurriendo cambios necesarios de tomar en cuenta en las supervisiones y negociaciones comerciales futuras. Las Figuras 4 y 5 , tomadas de un reciente trabajo elaborado por J. George, de Motorola, Inc. ${ }^{25}$, presentan graficamente lo que ha estado succdiendo con los productos y mercados de semiconductores. Tal como se puede apreciar en la Figura 4, en la actualidad las empresas japonesas

realizadas por la GAO en su Indexof reports and testimony: fiscal year 199I, que se puede solicitar bajo la dencminación de GAOFOPA91-15 a la U.S. General Accounting Office, PO Box 6015, Gaithersberg. MD 20887.

$25 /$ J. George, "Tligh technology competition between U.S. and japanese companies". EI señor George es vicepresidente corporativo y gerente general de la MOS Memory Products Division de Motorola, Ine. 


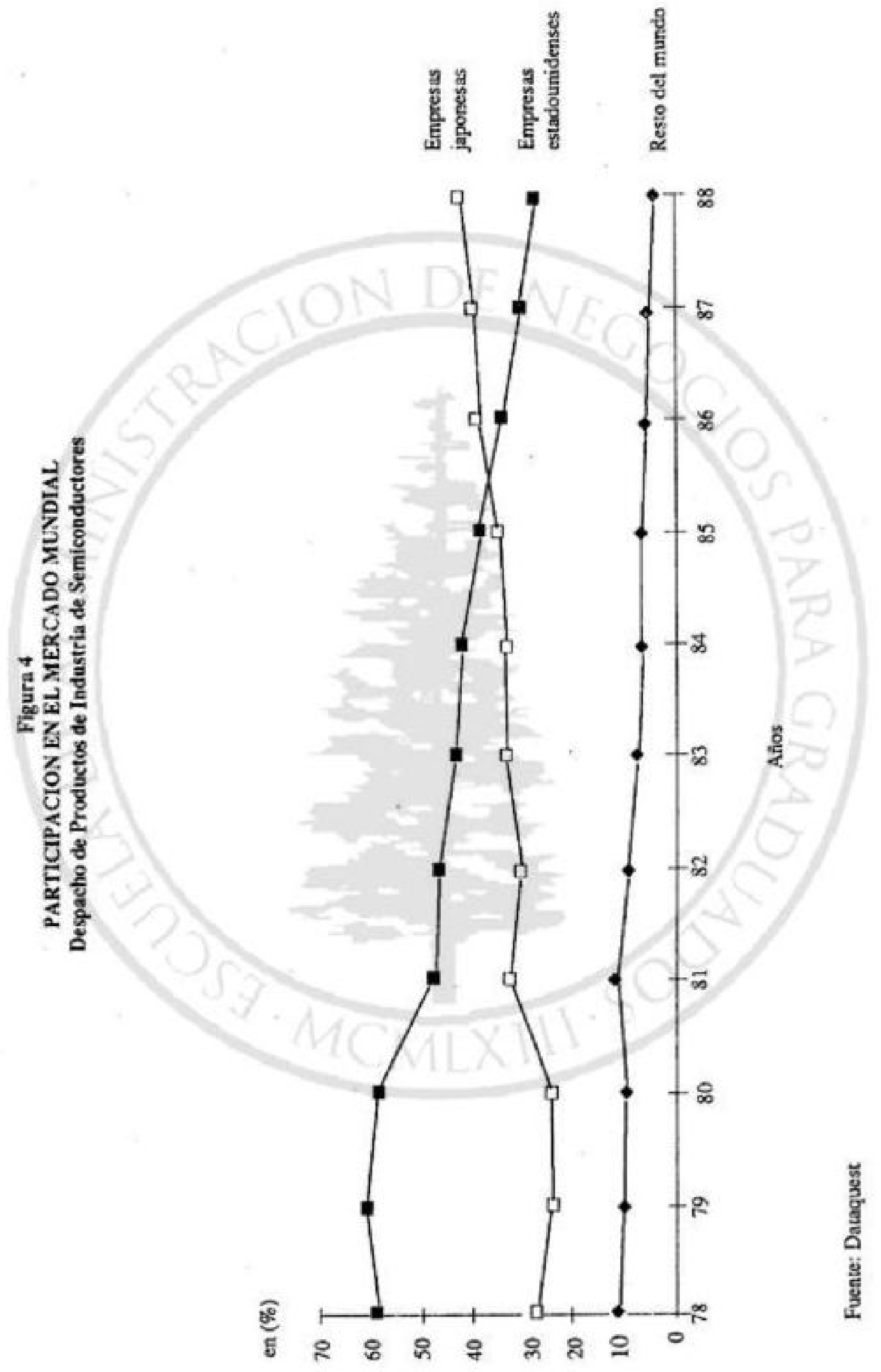




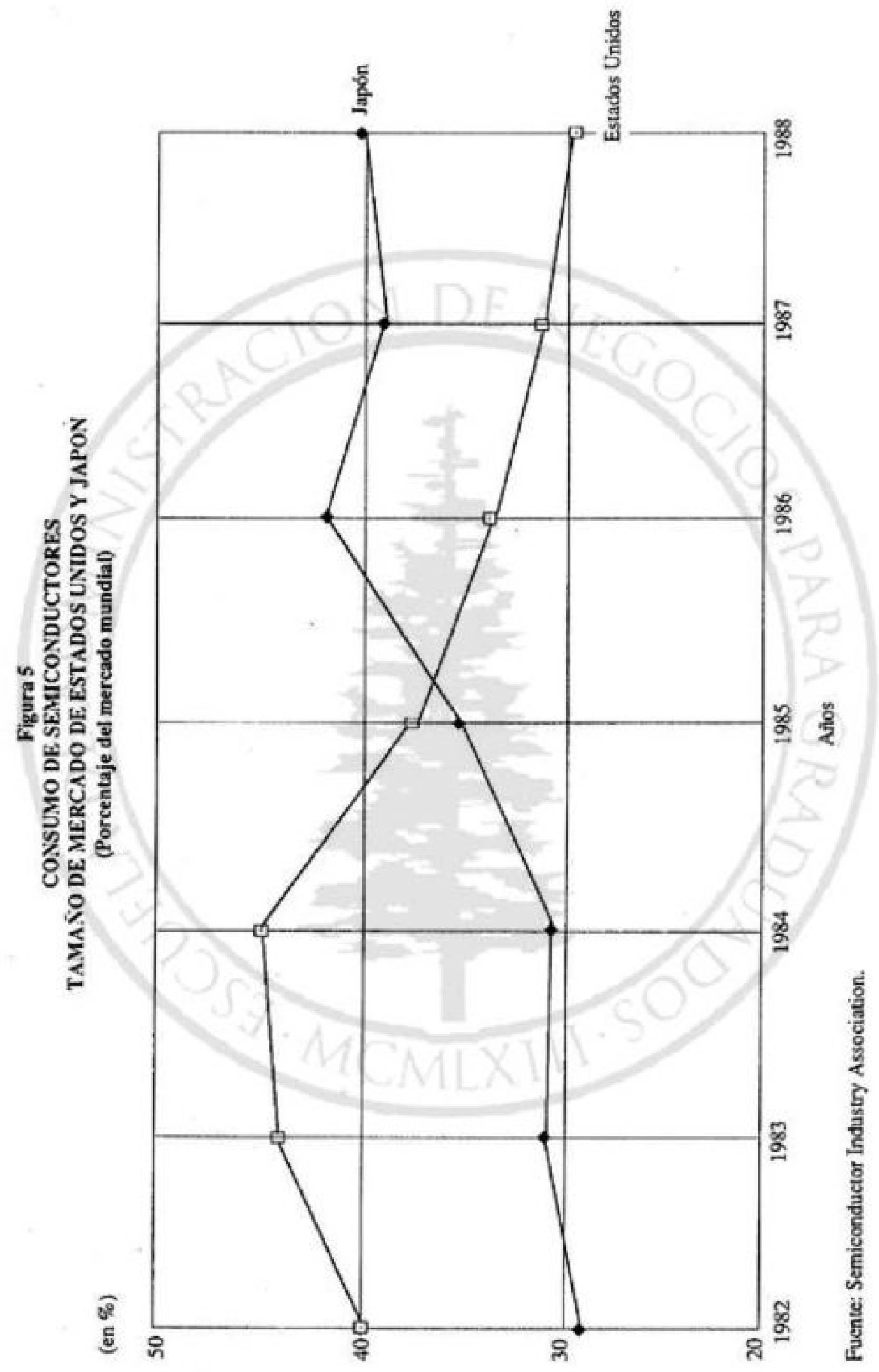


dominan $\mathrm{cl}$ mercado mundial, habiendo desplazado a Estados Unidos de esta posición, en términos de embarques, en algún momento entre 1985 y 1986 (de un máximo en 1984). Sin embargo, desde el punto de vista de la negociación de la política comercial, es más importante lo descrito en la Figura 5, que muestra que el Japón es el más grande mercado de semiconductores.

Esta situación explica por qué los japoneses han podido "mantenerse firmes" en las negociaciones con el Presidente Bush y suequipo. También muestra que cl trabajo de la Embajadora Carla Hill. Representante Comercial Especial de los Estados Unidos (quien no cra miembro de cste equipo) no funcionó en esta área. Con más del 40 por ciento del consumo mundial concentrado ahora en el Japón y menos del 30 por ciento en Estados Unidos, ella merece todo el apoyo que pueda conseguir. Jim Gcorge, de Motorola describe muy bien este estado de cosas:

"Basado en la experiencia de mi empresa, puedo decir que existen ciertas cosas bísicas que podemos hacer para aleaszar el éxito. Número uno, debemos tener acceso al mereado japonés. Sin eso, va a ser muy difícil competir en el negocio de semiconductores. El Japón es el mercado más grande del mundo y estál creciendo más rápidamente que los demás. Si se da por perdido el 40 por ciento del mereado mun. dial y nos concentramos en el 60 por ciento restante, las economias de escala comienzan en realidad a operar en nuestra contra $19 \%$,

Por supuesto, la "economía de escala" de la que habla George no se refiere a un producto único. En la industria de los semiconductores constantemente están cambiando los productos y los procesos. Esta expansión ha sido de vital importancia para otros productos tradicionalmente variables, tales como los automóviles, y también ha estimulado el desarrollo de una serie de productos nuevos. Estos incluyen alternativas a productos y procesos existentes, como los que están apareciendo en las cada vez mayores capacidades de los chips (y software) mejorados e incorporados en estaciones de trabajo relativamente pequeñas y económicas que pueden ser usadas en lugar de las procesadoras centrales y minicomputadoras más grandes y más costosas ${ }^{27 !}$. Es im. portante continuar con csta expansión de modo que siga produciendo cste tipo de bencficios. Este trabajo está dirigido a lograr estos fines. Las orientaciones enunciadas aquí tienen este propósito al propugnar que las cosas se muevan en la dirección correcta.

Tal como observamos en nucstra introducción, los montos comprometidos, por ejemplo, en las inversiones federales en investigación y desarrollo (I\&.D) son de por sí clevados. Las instituciones necesarias para llevar a caho lo que estamos sugiricndo ya existen y cucntan con personal experimentado y competente. Estas instituciones ofrecen una ventaja adicional, por cuanto lo que estamos sugiriendo no tiene por qué llamarse "politica industrial" si cso no es políticamente aceptable. Al trabajar con instituciones ya existentes, no se requiere de tiempo para reorientarlas a apoyar la transición de una economía de Gucrra Fría a una cconomía de tiempos de paz. Al suprimir el térmi-

27 Vease: "Cut rate computing". Business Week, Jan. 20, 1992. p. 30. 
no "política industrial" podemos, de ser necesario, refcrimos a esto como a un "dividendode paz". Este cambio de terminología pucde abrir también el camino para atender a los gastos (que podrían llamarse mejor "inversiones") necesarios en infracstructura. Las inversiones en escuelas, caminos, desarrollo urbano, etc., son reconocidas como responsabilidad del gobierno; de modo que en este caso ya existen también las instituciones adecuadas para ejecutar estos programas. 